\title{
COMPLEX SECURITY SYSTEM FOR PREMISES UNDER CONDITIONS OF LARGE VOLUME OF PASSENGER TRAFFIC
}

\author{
Vladimir Yakubov, Sergey Shipilov ${ }^{1}$, Grigoriy Parvatov, Vladimir Antipov, \\ Timur Muksunov \\ National Research Tomsk State University, the Department of Radiophysics, 634050 Tomsk, Russia
}

\begin{abstract}
Subsystems of the design of a complex security system for premises under conditions of large volume of passenger traffic are considered. These subsystems provide video- and thermal imaging control, radio wave tomography, and gas analysis. Simultaneous application of all examined variants will essentially increase the probability of timely prevention of dangerous situations with the probability of false alarm as low as possible. It is important that finally, this will provide protection of population and will facilitate the work of intelligence services.
\end{abstract}

\section{Introduction}

The urgency of the development of a complex security system in transport with intensive passenger traffic is obvious nowadays. Among the most important places that require special attention are entrances at railway stations, airports, and subway stations. A paramount problem here is real-time detection and neutralization of various items forbidden for transportation including fire and cold steel arms, explosives, and toxicants. Constantly increasing threat of terrorist attacks is a reality of modern life. Both luggage and passengers are inspected. Whereas x-ray apparatuses are suitable for luggage inspection, the problem is much more complicated for passenger inspection. Even low doses of x-ray radiation can be accumulated in multiple trips and can cause various diseases. The same is true for protection of intelligent service personnel from radiation exposure. However, the security problem is acute not only in transport, but also in protection of premises and territories of manufacture and storage of dangerous substances and their components, protection of banks and public institutions, places of mass events, as well as schools, high schools, etc. Nevertheless, transport remains the most vulnerable link in the chain of all problems under conditions of large volume of passenger traffic.

What are the basic requirements to security systems? First of all, the security system should operate remotely, but be selective. This means that danger should be detected as soon as possible, before it will be manifested itself. Furthermore, the system operation must be hidden - it should neither provoke potential terrorists nor create excessive inconveniences for ordinary passengers. The next requirement is that the system should

${ }^{1}$ Corresponding author: s.shipilov@gmail.com 
operate quickly, that is, detection should be carried out in real time and create no jams. Parallel inspection can be used here. Finally, there is the requirement of highly reliable danger detection, with reasonably low level of false alarms. This requirement leads inevitably to a multi-parametric system, that is, to a complex system when different physical methods of sensing are used simultaneously or sequentially [1-6].

\section{Video and thermal vision subsystems}

Video control is the first integral part of any complex security system. The complexity of this subsystem (Figure 1) is a huge flow of quickly changing information that must be analyzed using supercomputer technologies and special programs of face control by reference points. Anyway, a database of undesirable or potentially dangerous persons must be preliminary created here [4]. In this subsystem, an analysis of the dynamics of movements of suspected person limbs - hands and feet - and head can be used. This direction is now being intensively developed and promises great results. It is important to note that detection can be performed just outside the entrance to the premises being protected. Sufficient range of detection is $50 \mathrm{~m}$.
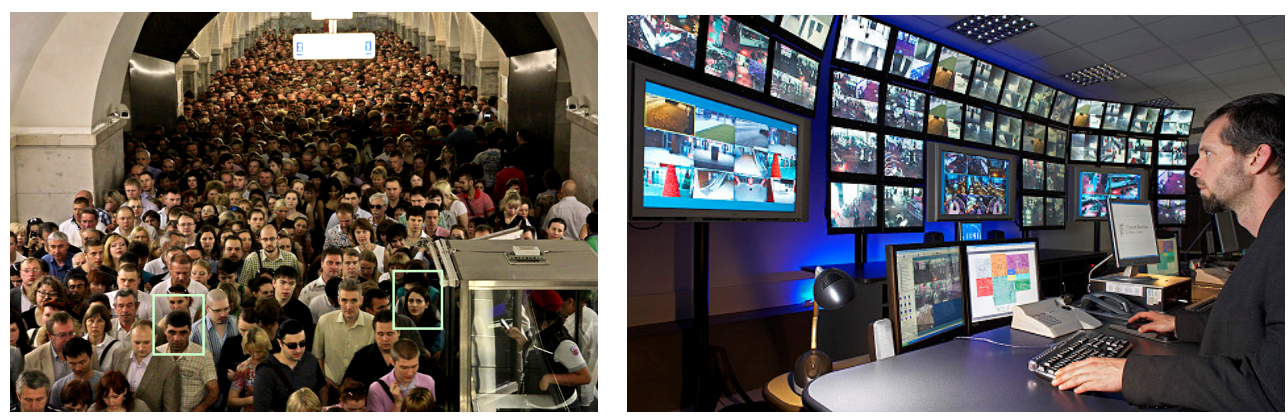

Figure 1. An example of operation of a system of video observation and identification of metro visitors.

The thermal vision subsystem allows potentially dangerous or forbidden items hidden under clothes to be identified (Figure 2). Here the contrast between the human body temperature and an extraneous item hidden under clothes is used. The maximal range of detection is $30 \mathrm{~m}$. The item is identified by its shape. Figure 2 shows an example of visualization of a cellular telephone under clothes using a thermal vision subsystem. 


\section{Doppler subsystem}

The Doppler subsystem additionally equipped with a focusing system is capable of detecting quickly moving people against the background of a quietly moving crowd. Narrow-band radio radiation, for example, at a frequency of $24 \mathrm{GHz}$ is used. It is suffice to perform spectral analysis of reflected signals. This subsystem is easily combined with the video and thermal vision subsystems. It is important that the Doppler subsystem can be used to record the relief of the radio image of a hidden item. Figure 3 shows the results of restoration of the image of a test object shaped as two metal plates with planes mutually shifted by approximately $4 \mathrm{~mm}$ and deviated from the vertical. The geometrical sizes of plates were $35 \times 15 \mathrm{~cm}$. This test object does not lie in one plane and imitates an object with a relief. It can be seen that the deviation of plates and their mutual shift in the image depth are unambiguously reconstructed.

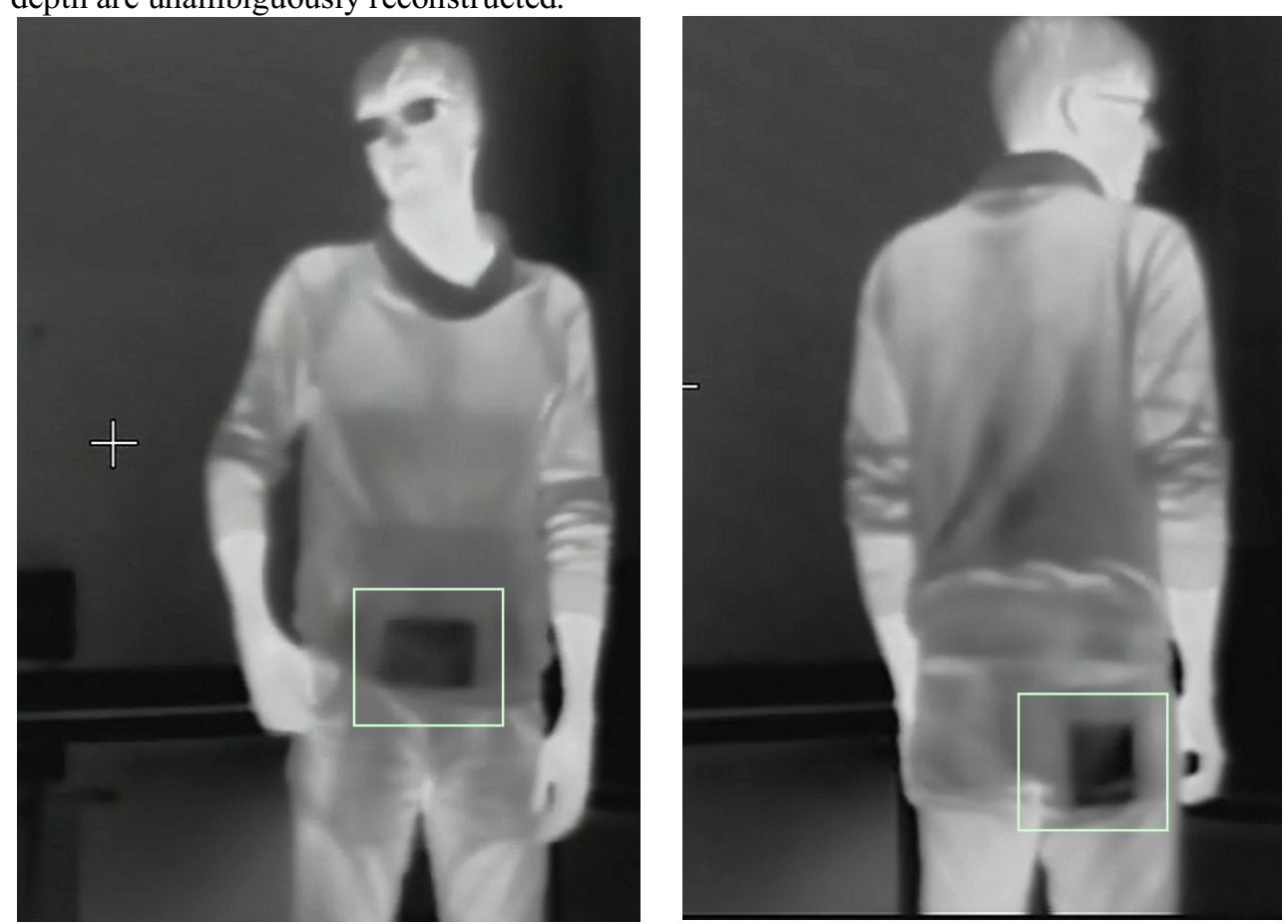

Figure 2. An example of thermal vision detection of an item hidden under clothes using the developed software. 


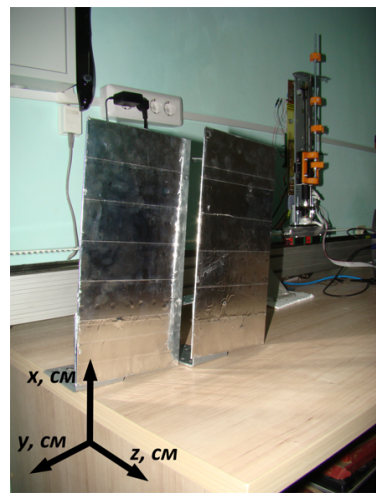

a

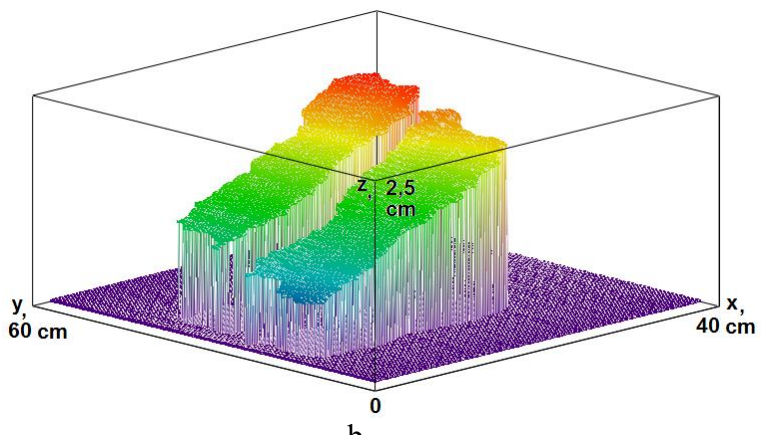

$\mathrm{b}$

Figure 3. Restoration of the relief of a test object: photograph (a) and reconstructed 3D relief (b).

The phase method of relief reconstruction was used. The concept is based on the idea of creation of a phased antenna array (PAA) of radio range with electronic control. According to this method, radiation is focused in a preset region of space, and subsequent scanning by the focus point over the entire examined area is performed. Radiation focusing can be implemented on the physical level - using lenses and mirrors - and on the computer level using the technology of wide aperture synthesis. A combination of these levels is the most perspective direction from the viewpoint of fast response, adaptability to manufacture, and commercial attractiveness. Figure 4 shows as a flat reflective aperture can focus radiation. It is suffice to create the corresponding distribution of the reflected signal phase, for example, using its binary values $\pm \pi$.
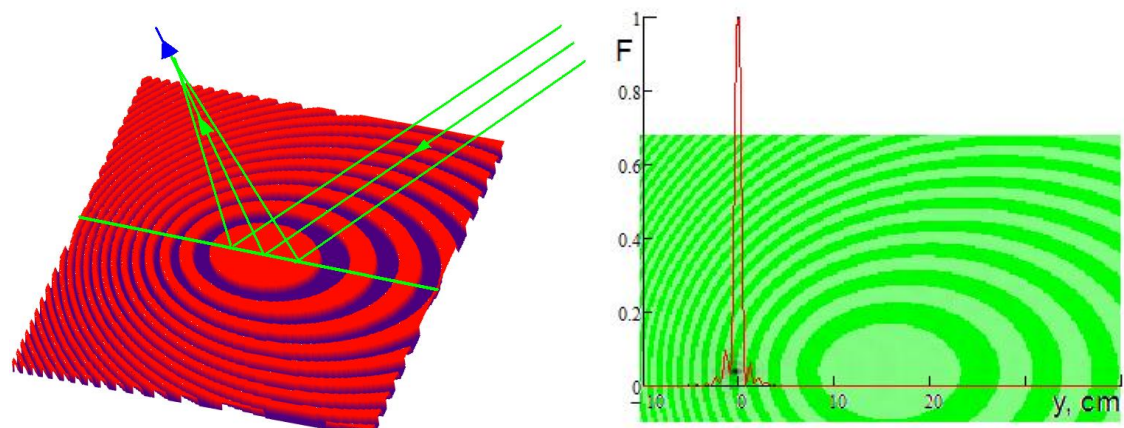

Figure 4. Radiation focusing with a flat reflector.

Fast switching of the collective reflector phase distribution will allow one to control effectively the direction of reflected radiation and hence to obtain the desired reflective properties. The formation of required distributions for any base surface allows methods of radio tomography to be used to develop the technology of the so-called "Clever Skin."

The suggested technology based on reflective PAA is not exotic. This principle has already been declared in a tomography Smith Detection's eqo people screener (USA - Jürgen Sachs -Handbook of Ultra-Wideband Short-Range Sensing: Theory, Sensors, Applications - Wiley-VCH -2012). Its external view is shown in Figure 5. In modern radiolocation, reflecting and transmitting waveguide-type antenna arrays have already been used. 


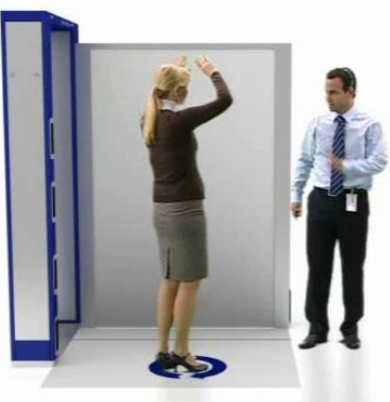

a

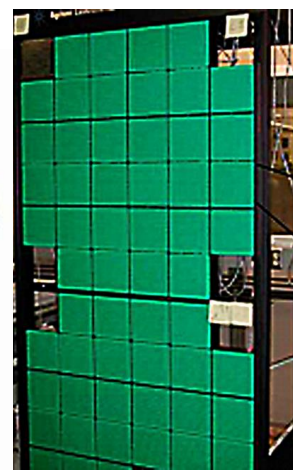

b

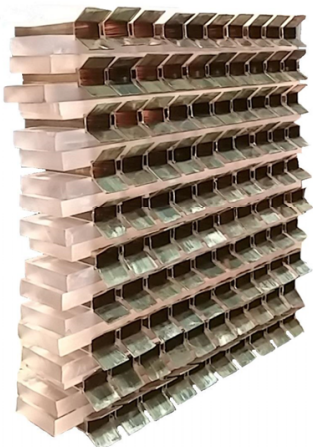

$\mathrm{C}$

Figure 5. Tomography Smith Detection's eqo people screener (USA) with reflective PAA (a), panel from reflective elements (b), and controllable reflecting waveguide-type unit developed at TSU (c).

\section{Ultra-wideband subsystem}

The next subsystem that deserves special attention is also the radio-wave ultra-wideband (UWB) subsystem being in its essence a radio-wave tomograph (Figure 6). This subsystem uses ultrashort sensing impulses, a clocked switchable linear UWB transceiving antenna array, and technology of aperture synthesis for signals processing. This radio-wave tomograph allows one to visualize movement of people behind barriers or through walls. The attained video-image change rate was 10 frames/s. This makes it possible to estimate the emotional state of a person from its breathing frequency.

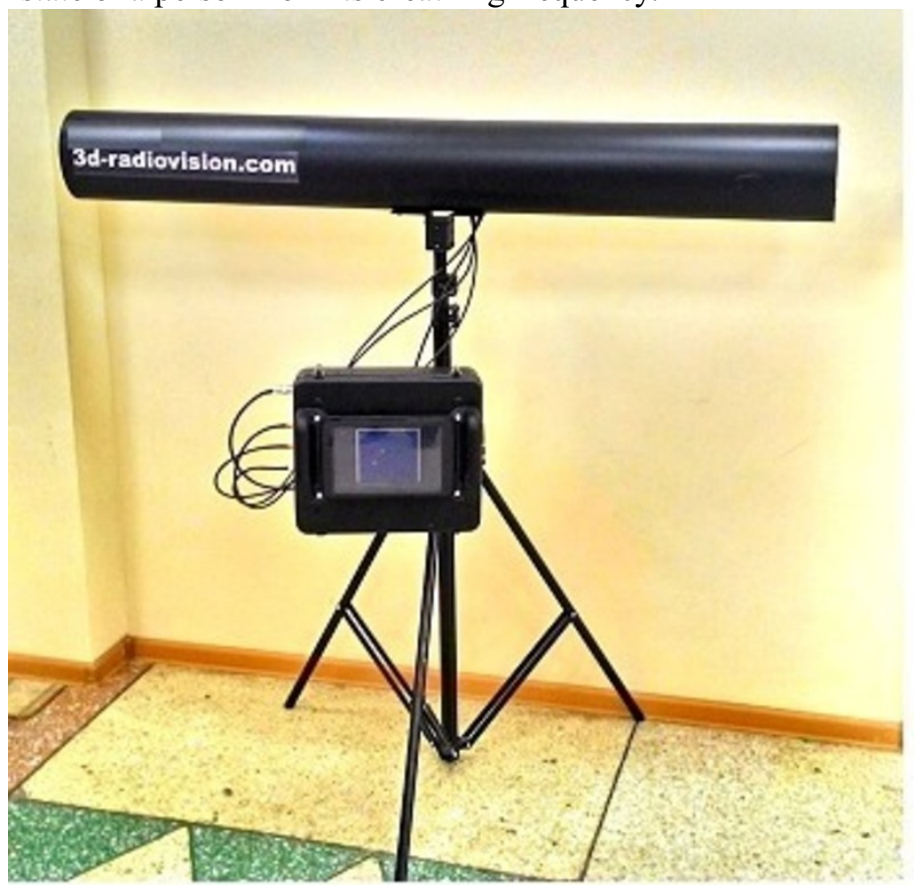

Figure 6. Linear radio-wave tomograph "Radiodozor" intended to control movement of people through barriers. 
Figure 7 shows an example of possible application of this subsystem in conducting an anti-terrorist operation.

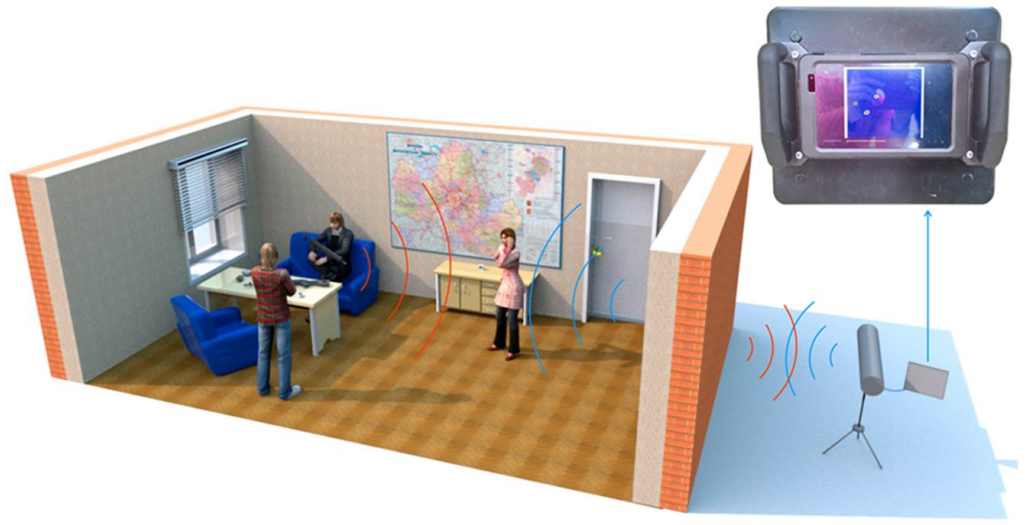

Figure 7. An example of possible application of "Radiodozor" to conduct an anti-terrorist operation.

The next device of the tomographic type is a portable one intended for real-time and safe inspection of items forgotten in transport. Figure 8 shows an example of radio-wave tomography of 4 coins hidden in a box located behind a wall. It can be seen in which layer these coins are located. The device represents an UWB radio-wave tomograph with a planar clocked antenna array. The time of measuring and recording the complete $3 \mathrm{D}$ tomogram is $8 \mathrm{~s}$.

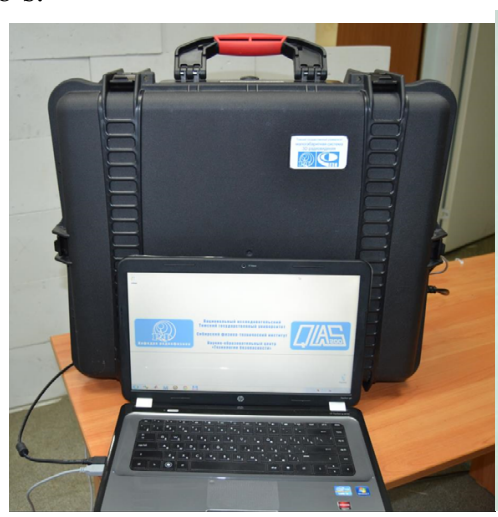

a

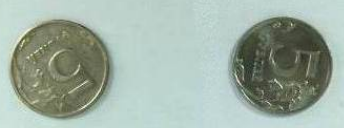

$\mathrm{b}$

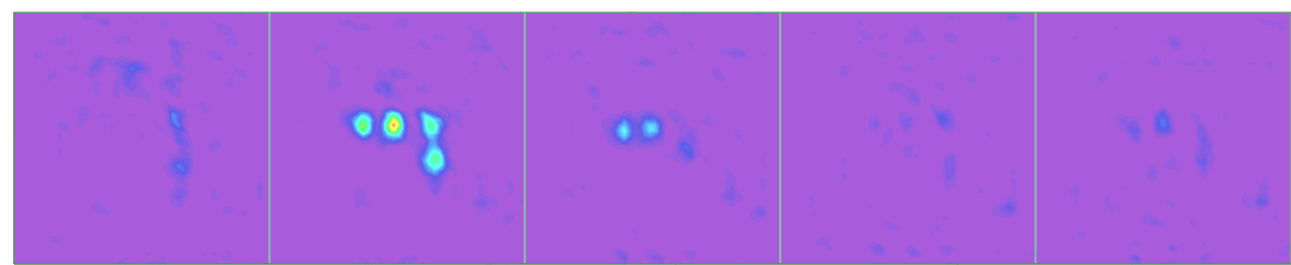

c

Figure 8. Portable UWB radio-wave tomograph (a), hidden test object in the form of coins (b), and its radio-wave tomogram $(\mathrm{c})$. 


\section{Nonlinear radar subsystem}

The nonlinear radar subsystem (Figure 9) is sufficiently promising for security provision. It allows hidden radio electronic devices (cellular telephones, detonators, transmitters, miniature video cameras, taps etc.), detonators, and killing metal elements to be detected on a human body or in a hand luggage. To increase the information content, the radar is capable to conduct additionally zonal inspection of a person. This makes it possible to localize radio engineering devices on a human body and to estimate the degree of their danger (Figure 9). The range of action of the radar is $10-25 \mathrm{~m}$.

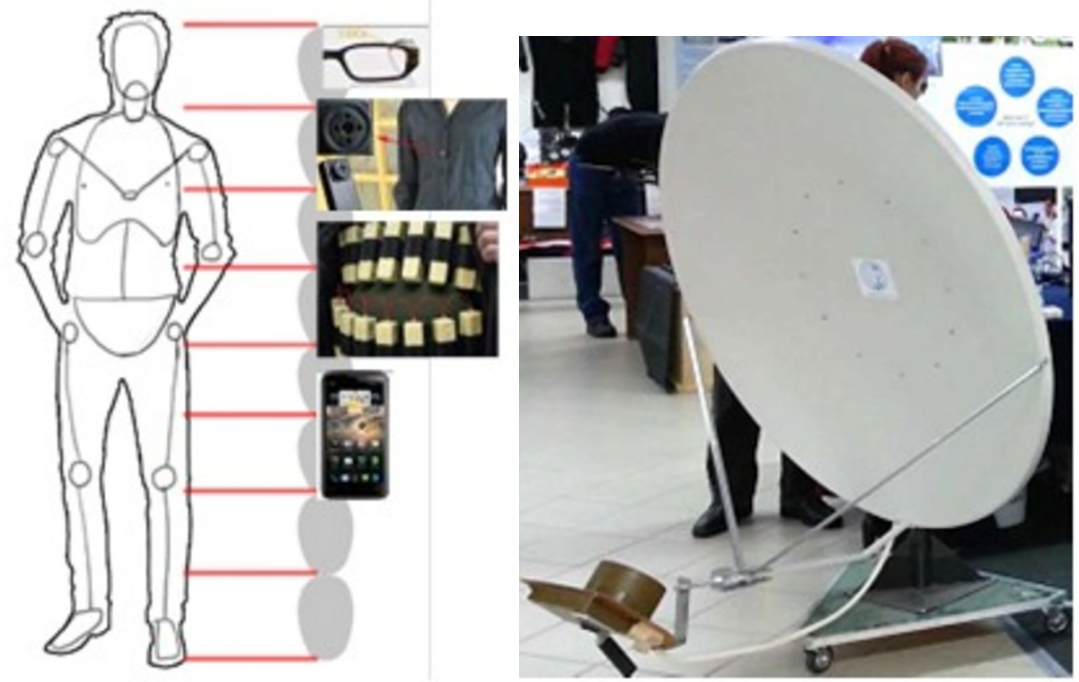

Figure 9. Remote detector developed to detect hidden radio electronic devices.

The next security subsystem is a multi-sensor system of highly sensitive semiconductor gas sensors (Figure 10) that allows ultra-low concentrations of explosives and toxicants to be detected in air and a source (carrier) of dangerous substances to be identified (Figure 11) [7]. Components of the system can be mounted in the ceiling and can be invisible to visitors (Figure 12).
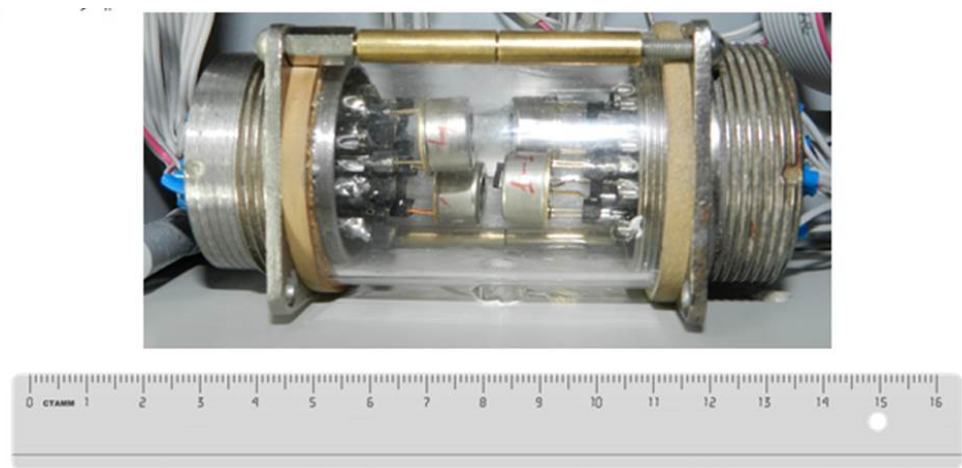

Figure 10. Multisensor developed for detection of explosives and toxicants. 


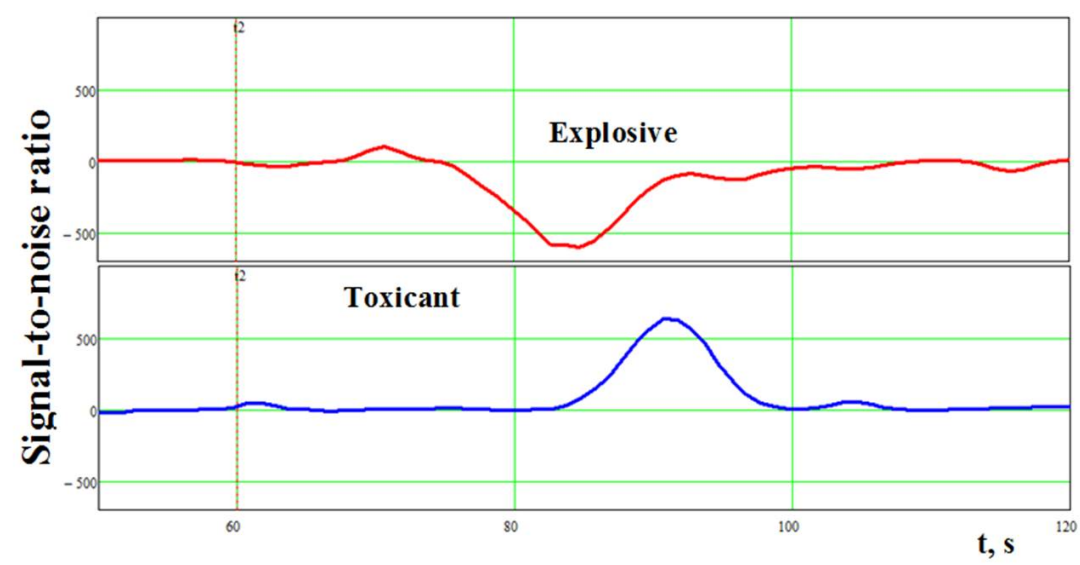

Figure 11. Multisensor response to small concentrations of explosives and toxicants.

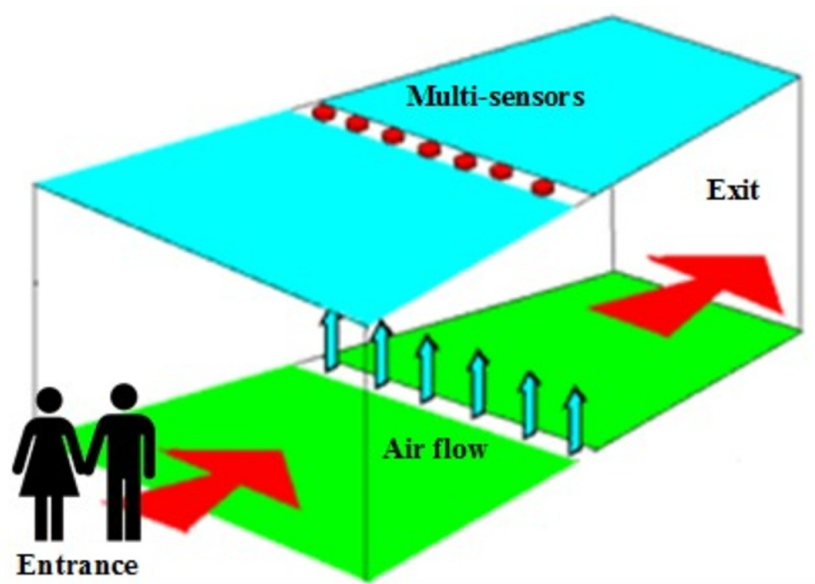

Figure 12. Example of arrangement in the ceiling of the entrance of a premise of gas multi-sensors intended for detection traces of explosives and toxicants on visitors.

\section{Conclusion}

The subsystems considered above provide the basis for the complex security system for premises under conditions of a large volume of passenger traffic. Combined application of all subsystems will essentially increase the probability of timely prevention of dangerous situations with the probability of false alarm as low as is wished. Finally, this ensures the protection of people and facilitates the work of intelligent services.

\section{Acknowledgment}

This research is conducted as part of the Program of Scientific Foundation n. a. D.I. Mendeleev in Tomsk State University (Project No. 8.2.48.2015). 


\section{References}

[1] V.P. Yakubov, S.E. Shipilov, R.N. Satarov, A.V. Yurchenko, Technical Physics. The Russ. J. of Applied Phys. 60, 279 (2015) doi: 10.1134/S1063784215020267

[2] S. Kharkovsky, R. Zoughi, IEEE Instrum. Meas. Mag. 10, 26 (2007) doi:10.1109/MIM.2007.364985

[3] Y. Kan, Y. Zhu, Q. Fu, MATEC Web of Conferences 56, 02001 (2016) doi10.1051/matecconf/20165602001

[4] L. Lianlin, W. Zhang, F. Li, IEEE Trans. Geosci. Remote Sens. E 48, 415 (2010) doi: 10.1109/TGRS.2009.2024690

[5] Tan Guo, Xiao Heng Tan, Chao Chen Xie, MATEC Web of Conferences 44, 01039 (2016) doi: 10.1051/matecconf/20164401039

[6] Gregory J. Mazzaro, F. Martone Anthony, M. McNamara, IEEE Transactions on Aerospace and Electronic Systems 50, $477 \quad$ (2014) doi:10.1109/TAES.2013.120798

[7] T.R. Muksunov, N.K. Maksimova, E.J. Sevast'anov, S.E. Shipilov, V.P. Yakubov, Russ. Phys. J. 57, 1287 (2015) doi: 10.1007/s11182-015-0376-1 